\title{
Die zwei Seiten des Frühlings
}

E s wird Frühling - nicht nur kalendarisch, sondern auch für unsere Patienten mit sekundär wie primär chronisch-progredienter MS. Während für Patienten mit schubförmig verlaufender MS in den letzten Jahren ein neues Medikament das nächste jagte, hatten wir jenen mit chronischprogredienten Verlaufsformen relativ wenig anzubieten. Das ändert sich erfreulicherweise: Das im September 2016 zugelassene Daclizumab erhielt von der EMA ein so breites Label, dass auch Patienten mit sekundär chronisch-progredienter MS und noch vorhandener Schubaktivität behandelt werden können. Die Auswertung der Siponimod-Studie ergab, dass die Substanz bei Patienten mit sekundär chronisch-progredienter MS die Progression bremst. Und nun zeigte auch die ORATORIO-Studie, dass Ocrelizumab bei Patienten mit primär chronischprogredienter MS im Vergleich zu Placebo die Erkrankungsprogression signifikant reduziert.

Das ist alles sehr erfreulich. Aber der Frühling hat eben nicht nur warme und sonnige Tage im Mai und Juni, sondern auch sehr durchwachsenes Wetter im März und April. Daher sollten wir bei aller Freude über die besseren Behandlungsmöglichkeiten nicht in blinde Euphorie verfallen und unerfüllbare Erwartungen wecken. Denn bei genauer Betrachtung fällt ein wichtiger Aspekt auf: Es profitieren insbesondere die jüngeren Patienten, die trotz chronisch-progredientem Verlauf noch unter inflammatorischer Aktivität leiden. Mit anderen Worten: Der ältere Patient, der seit 20 Jahren unter einer primär chronisch-progredienten MS leidet und seit zehn Jahren weder einen neuen noch einen aktiven Herd im MRT zeigt, wird sehr wahrscheinlich kaum von den neuen Medikamenten profitieren. Hier werden wir wohl die eine oder andere Hoffnung, die jetzt durch Berichte in den Medien induziert wird, enttäuschen müssen. Man darf daher gespannt sein, wie die EMA den Zulassungstext für Ocrelizumab zur Behandlung der PPMS formulieren wird - und entweder eine breite uneingeschränkte Anwendung zulässt oder doch spezifi- sche klinische Kriterien vorsieht. Wichtig bleibt daher eine realistische und sachgerechte Besprechung der neuen Substanzen vorzunehmen, um nicht unrealistische Hoffnungen $\mathrm{zu}$ generieren. Die gute Nachricht ist aber, dass alle neuen Substanzen gut verträglich sind und ein gutes Sicherheitsprofil aufweisen, sodass wir uns in weniger eindeutigen Fällen guten Herzens im Sinne von in „dubio pro Therapie" entscheiden können.

Der Frühling kommt mit sonnigen Tagen außerdem noch zu einer Medikamentengruppe, mit der Neurologen und Psychiater bisher weniger zu tun hatten: den Biosimilars. Den Startschuss gab das erste Biosimilar für Glatirameracetat $\left(\mathrm{Clift}^{\varpi}\right)$, das jetzt hierzulande auf den Markt gekommen ist. Das Besondere an den Biosimilars ist, dass eine reine Bioäquivalenzprüfung wie bei den Generika nicht ausreicht, sondern für eine Zulassung teils auch klinische Studiendaten notwendig sind. Genau diese Daten hat der Hersteller nun vorgelegt und zeigen können, dass keine Unterschiede hinsichtlich Wirksamkeit und Verträglichkeit zwischen dem Original und dem Biosimilar nachweisbar sind. Es geht aber weiter: Nun kommt auch das erste Biosimilar für Rituximab auf den Markt. Allerdings wurde hier noch keine klinische Studie vorgelegt, so dass eine Zulassung für die Behandlung der MS derzeit (noch) nicht ansteht. Jedoch haben bereits zwei weitere Firmen angekündigt, Rituximab-Biosimilar auf den Markt bringen und sich alle denkbaren Indikationen anschauen zu wollen. Für die Originalhersteller B-Zell-depletierender monoklonaler Antikörper, insbesondere auch für den OcrelizumabHersteller, könnten daher schon im Herbst Unwetter in Orkanstärke aufziehen. In den USA ist bereits eine heftige Diskussion über den Nutzen von Biosimilars in der Behandlung der MS ausgebrochen, diese steht uns noch bevor. Genießen Sie bis dahin die sonnigen Frühlingstage!

Ihr

Volker Limmroth 